\title{
Cap'n'collar differentiates the mandible from the maxilla in the beetle Tribolium castaneum
}

\author{
Joshua F Coulcher and Maximilian J Telford ${ }^{*}$
}

\begin{abstract}
Background: The biting mandible of the arthropods is thought to have evolved in the ancestor of the insects, crustaceans and myriapods: the Mandibulata. A unique origin suggests a common set of developmental genes will be required to pattern the mandible in different arthropods. To date we have functional studies on patterning of the mandibular segment of Drosophila melanogaster showing in particular the effects of the gene cap'n'collar (cnc), however, the dipteran head is far from representative of insects or of more distantly related mandibulates; Drosophila does not even possess a mandibular appendage. To study the development of a more representative insect mandible, we chose the red flour beetle Tribolium castaneum and investigated the function of the Tribolium orthologs of cap'n'collar (Tc-cnc) and the Hox gene Deformed (Tc-Dfd). In order to determine the function of Tc-cnc and Tc-Dfd, transcripts were knocked down by maternal RNA interference (RNAi). The effects of gene knockdown were examined in the developing embryos and larvae. The effect of Tc-cnc and Tc-Dfd knockdown on the expression of other genes was determined by using in situ hybridization on Tribolium embryos.
\end{abstract}

Results: Our analyses show that Tc-cnc is required for specification of the identity of the mandibular segment of Tribolium and differentiates the mandible from maxillary identity. Loss of Tc-cnc function results in a transformation of the mandible to maxillary identity as well as deletion of the labrum. Tc-Dfd and the Tribolium homolog of proboscipedia ( $T c-m \times p=$ maxillopedia), Hox genes that are required to pattern the maxillary appendage, are expressed in a maxilla-like manner in the transformed mandible. Tribolium homologs of paired (Tc-prd) and Distal-less (Tc-DII) that are expressed in the endites and telopodites of embryonic appendages are also expressed in a maxilla-like manner in the transformed mandible.

We also show that Tc-Dfd is required to activate the collar of Tc-cnc expression in the mandibular segment but not the cap expression in the labrum. Tc-Dfd is also required for the activation of Tc-prd in the endites of the mandible and maxillary appendages.

Conclusions: $T c-c n c$ is necessary for patterning the mandibular segment of Tribolium. Together, Tc-cnc and Tc-Dfd cooperate to specify mandibular identity, as in Drosophila. Expression patterns of the homologs of $c n c$ and Dfd are conserved in mandibulate arthropods suggesting that the mandible specifying function of cnc is likely to be conserved across the mandibulate arthropods.

Keywords: Beetle, cap'n'collar, Deformed, Endite, Labrum, Mandible, Maxilla, RNAi, Tribolium

\footnotetext{
* Correspondence: m.telford@ucl.ac.uk

Department of Genetics, Environment and Evolution, University College

London, Darwin Building, Gower Street, London WC1E 6BT, UK
} 


\section{Background}

The arthropod mandible is an appendage adapted for biting and chewing and is present in three arthropod groups, the insects and crustaceans (collectively the Pancrustacea) and the myriapods (millipedes and centipedes). The mandibulate arthropods, commonly grouped together in the monophyletic Mandibulata, constitute the majority of animals both in terms of numbers of species and biomass on this planet. The mandible is therefore an evolutionary novelty of particular interest.

There are many different types of mandible, but the characteristic that most mandibles share, and which differentiates it from other arthropod appendages, is the presence of a functional biting edge made up of the incisor and molar processes. This gnathal edge is widely considered to be a homologous structure within the Mandibulata [1-3].

Other arthropod groups, the chelicerates and trilobites, do not have mandibles and instead have a walking leg on the homologous segment to the mandibular segment $[4,5]$. An unsegmented appendage, or lobopod, is present in closely related outgroups to the arthropods, such as the onychophorans and tardigrades [6].

An alternative phylogenetic hypothesis to the monophyletic Mandibulata is the Myriochelata hypothesis, which groups the myriapods with the chelicerates. Accepting this hypothesis would suggest that the mandible evolved independently in the Myriapoda and Pancrustacea or that it has reverted to a walking leg in the Chelicerata [7]. While still controversial, recent molecular phylogenies including evidence from unique microRNAs favour Mandibulata over Myriochelata. This phylogeny is also strongly supported on morphological grounds [8-11].

\section{Mandible evolution}

The mandible is serially homologous with other arthropod post-antennal appendages all of which are thought to have evolved from a segmented biramous limb. The archetypal biramous limb consists of a protopodite (the base of the limb) to which are attached two branches: the telopodite (or palp) and an exopodite [12-14]. Structures called endites, often involved in food processing, are also present on the protopodite. The gnathal edge of the mandible is thought to have evolved from the proximal most endite on the protopodite of this ancestral biramous limb $[2,11]$. The mandible is thought to be a gnathobasic structure and this interpretation is supported by expression data: the distal limb expression domain of Distal-less (Dll) is missing from the embryonic mandibular limb bud in diverse mandibulate arthropods [15-17].

All arthropod mandibles appear to be gnathobasic and are restricted to a monophyletic group implying that the mandible has a unique origin and is a homologous structure between mandibulate arthropods. We might therefore expect significant similarities in the embryonic patterning of the mandible between diverse mandibulate taxa. Finding the identity of the genes that pattern the mandible and showing how they function in diverse arthropod taxa could support the view that the mandible is homologous across the Mandibulata and, through comparisons with non-mandibulate sister groups, could give an insight into how the mandible evolved from a primitive arthropod limb.

We have undertaken a functional study of some of the genes that pattern the mandible in a model organism with a typical insect mandible to compare its development with the development of mandibles in other taxa. We chose to study the red flour beetle Tribolium castaneum that, unlike Drosophila melanogaster, has a canonical mandible in which the gnathal edge is made up of the incisor and molar processes.

\section{Mandibular segment patterning in Drosophila}

The majority of research into the function of genes patterning arthropod gnathal appendages has focused on insects with very derived mouthparts, in particular the involuted larval head and non-biting adult proboscis of the dipteran D. melanogaster [18-25] and the stylet of the hemipteran Oncopeltus fasciatus [26,27].

Although developing Drosophila embryos possess gnathal lobes (structures from which the gnathal appendages are formed in other less derived insects [28]), following head involution, Drosophila larvae do not have any gnathal appendages [28-31] and both larval and adult Drosophila lack an appendage on the mandibular segment.

In Drosophila, the gene Deformed ( $D f d$ ) is required for the specification of both mandibular and maxillary identities [23-25,32,33]. Dfd does not differentiate the mandibular segment from the maxillary segment; for this function another gene, cap'n'collar (cnc), is required [22-24]. cnc is a basic leucine zipper family gene (bZIP) that is expressed in an anterior 'cap' domain in the labrum and a posterior 'collar' domain in the mandibular segment and is necessary for the development of both labral and mandibular derived structures. It is likely that $c n c$ achieves its mandible patterning function in part indirectly by repressing the maxilla patterning function of $D f d$ : $D f d$ expression is repressed by cnc in the anterior of the mandibular gnathal lobe and the activity of the Dfd protein is also repressed by $c n c$ in the mandibular segment. $c n c$ null mutants lose both labral and mandibular segment derived structures and have a duplication of maxillary structures [22-24,34].

\section{Previous work in Tribolium}

In Tribolium, Brown et al. have demonstrated that the homolog of $D f d, T c-D f d$, is necessary for patterning the 
mandibular and maxillary segments and that $T c-D f d$ expression is progressively downregulated in the mandibular limb buds as in Drosophila [35,36]. In Tc-Dfd mutants there is a homeotic transformation of the mandible to an antenna and a loss of the maxillary endites. $D f d$, although required for mandible development, does not differentiate the mandibular segment from the maxillary segment in Drosophila or Tribolium. The role of Tc-cnc in Tribolium is not known, however, it is expressed in a very similar pattern to that seen in Drosophila [37] and this is also true of $c n c$ in other mandibulate arthropods [38-40] suggesting it may have a conserved function. Embryonic expression in non-mandibulate arthropods is not known.

\section{Experimental outline}

With the ultimate aim of understanding the origin of the mandible, we were interested in the role that Tc-cnc might play in patterning the mandibular segment of Tribolium castaneum, a mandible-bearing insect. In order to test its function in Tribolium, Tc-cnc was knocked down using parental RNA interference (RNAi) by injecting double-stranded RNA (dsRNA) into female Tribolium pupae [41]. The knockdown phenotype was detected both in embryos and in the first instar larvae of offspring of injected parents. The effect of Tc-cnc knockdown on downstream genes was studied by in situ hybridization in Tribolium embryos.

\section{Methods}

\section{Tribolium castaneum culture}

Wild-type T. castaneum (San Bernardino strain) were kindly provided by Dr Gregor Bucher (Department of Developmental Biology, Georg-August-University Göttingen, Göttingen, Germany) and raised at $32^{\circ} \mathrm{C}$ in organic wholemeal flour supplemented with $5 \%$ brewer's yeast.

\section{Cloning of Tribolium orthologs}

Tc-cnc, Tc-Dfd, Maxillopedia the Tribolium ortholog of Proboscipedia $(P b)(T c-m x p)$, the Tribolium ortholog of paired $(T c-p r d)$ and $T c-D l l$ were amplified from mixed stage cDNA by polymerase chain reaction (PCR) amplification using the following primers: $T c-c n c$, a 2,612 bp clone for haptenlabelled RNA probe synthesis (forward: 5'-GCAACAGTG

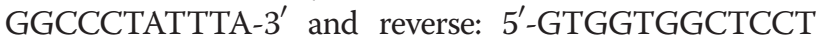
TGTGTTCT-3'). Tc-cnc, a 633 bp clone for dsRNA synthesis (forward: 5'-GATTACAGCTATACGAGTCGG-3' and reverse: $5^{\prime}$-GTCAGCCAGACTCAAAATCTG-3'). Tc-Dfd (forward: 5'-CCAAGTGAGGAGTACAACCAG-3' and reverse: $5^{\prime}$-TACAAGGCCGTGAGTCCGTAA-3'), Tc-mxp (forward: 5'-ATAGCTGCTTCGCTAGACCTTA-3' and reverse: $5^{\prime}$-TCGCAGGTGGGGTCATTAT-3'), Tc-Dll (forward: 5/-CAGCAGGTGCTCAATGTGTT- ${ }^{\prime}$ and reverse: 5'ATTAAACAGCTGGCCACACC-3'), Tc-prd (forward: $5^{\prime}$-ATGCACAGACATTGCTTTGG-3' ${ }^{\prime}$ and reverse: $5^{\prime}$ -
GGATCGTCACAGTGTTGGTG-3'). Accession numbers are as follows: Tc-cnc (GenBank: NM_001170642.1), Tc-Dfd (GenBank: NM_001039421), Tc-mxp (GenBank: NM_001114335), Tc-Dll (GenBank: NM_001039439), Tc-prd (GenBank: NM_001077622).

\section{Parental RNAi}

Parental RNAi was performed as previously described [41]: 0.25 to $0.4 \mu \mathrm{l}$ of $T c-c n c$ dsRNA (dissolved in distilled water at a concentration of 0.36 to $3 \mu \mathrm{g} / \mu \mathrm{l}$ ) was injected into female pupae. Then, 633 bp of Tc-cnc dsRNA (positions 1,389 to 2,021, including part of the bZIP domain which starts at position 1,932) was injected. Embryos were either fixed 24 to $48 \mathrm{~h}$ after egg laying or left to develop into first instar larvae for cuticle preparation. In total, 1,736 female beetle pupae were injected for collecting embryos for in situ hybridization.

In order to characterize the Tc-cnc phenotype, $218 \mathrm{fe}$ male pupae were injected with 1 to $2 \mu \mathrm{g} / \mu \mathrm{l}$ dsRNA and the cuticles of first instar larvae were analyzed. Of these 218 injected pupae, 195 successfully eclosed. At 20 days after injection a further 117 beetles (60\%) had died. Parental injection of Tc-cnc dsRNA resulted in the mortality of a much greater number of injected females compared to the numbers killed in other RNAi experiments, in which typically $10 \%$ of injected female beetles die by day 20 (data not shown). The higher mortality rate may be a consequence of the effects of Tc-cnc knockdown. Only one phenotype was detected in first instar larvae: transformation of the mandible to maxillary identity and loss of the labrum.

In order to obtain partial phenotypes (incomplete transformations of the mandible to maxillary identity) we tried injecting lower concentrations of $T c-c n c$ dsRNA (360 to $750 \mathrm{ng} / \mu \mathrm{l}$ ). However, only wild-type larvae or those with fully transformed mandibles were obtained, and no partial phenotypes were observed. Similar rates of mortality were observed even at lower concentrations.

To obtain $T c-D f d^{R N A i}$ embryos, 1,142 bp (positions 491 to 1,632$) T c-D f d$ dsRNA was injected into female pupae and embryos were fixed for in situ hybridization. The $T c-D f d^{R N A i}$ phenotype was confirmed by comparing cuticle preparations of first instar larvae to previously described phenotypes [36,42].

\section{Cuticle preparation}

Cuticles from first instar larvae were prepared in Hoyer's medium and lactic acid as previously described [43]. The cuticle preparations were observed using differential interference contrast (DIC) and confocal fluorescent microscopy (larval cuticle autofluoresces at visible wavelengths). Cuticle preparations were observed using confocal microscopy with an excitation frequency 
of $488 \mathrm{~nm}$ using an upright Leica TCS SPE confocal microscope (Leica microsystems, Wetzlar, Germany). Images were obtained and edited using Leica application suite advanced fluorescence software, LAS-AF (Leica microsystems, Wetzlar, Germany).

\section{Whole mount in situ hybridization}

Embryos were fixed in 9\% formaldehyde. Both single stainings (nitro blue tetrazolium/5-bromo-4-chloro-3-indolyl phosphate (NBT/BCIP)) and double stainings (NBT/BCIP and FastRed) were performed as previously described [44].
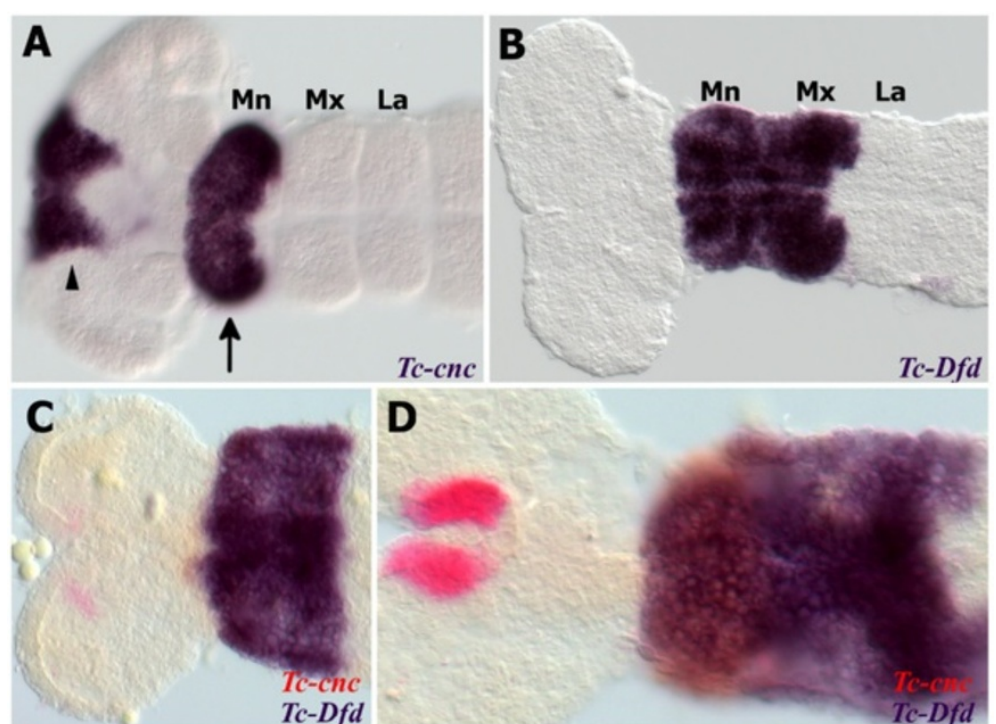

D
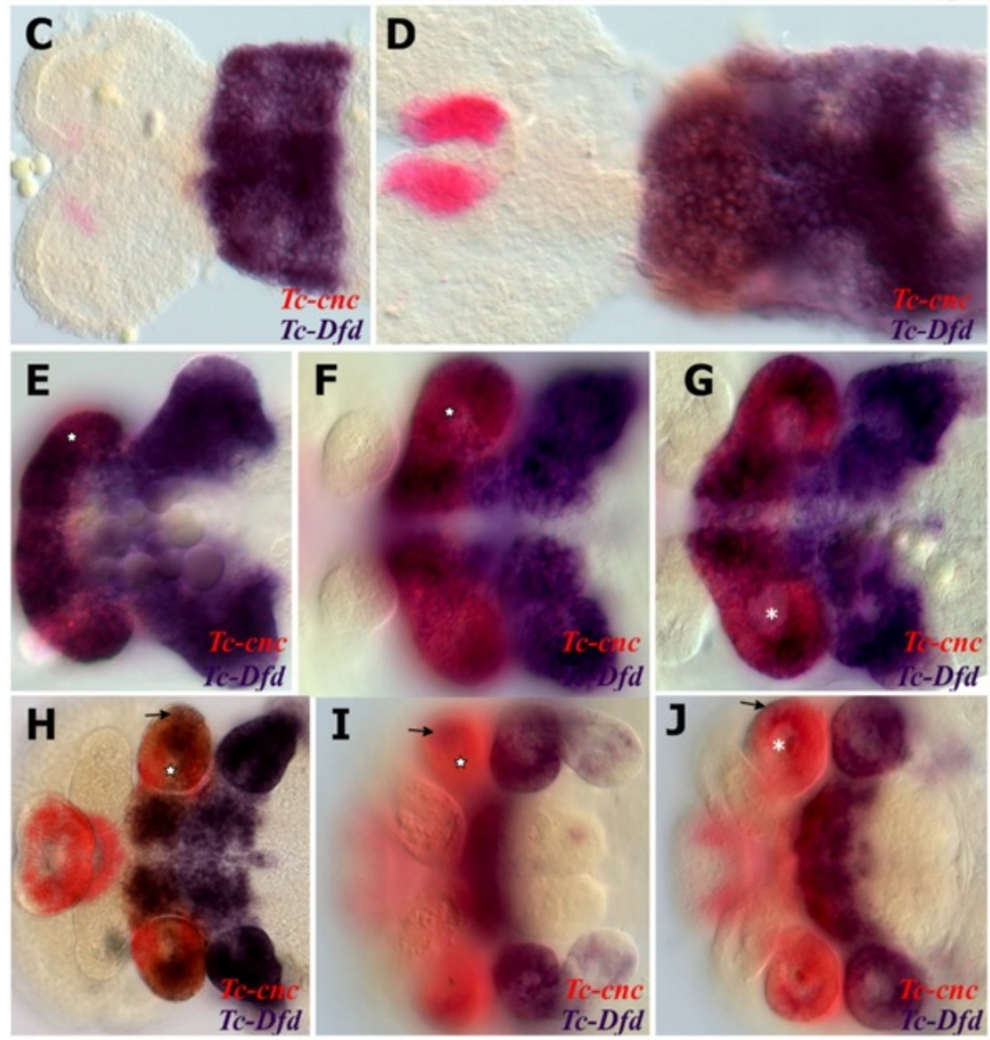

Figure 1 Expression of $T c-D f d$ and $T c-c n c$ in the mandibular and maxillary segments. All views are ventral with anterior to the left unless otherwise indicated. Gene expression was determined by in situ hybridization. (A) Expression of Tc-cnc in a germ band extending stage embryo. There is an anterior cap domain of Tc-cnc in the labrum (arrowhead). The posterior collar domain is present in the mandibular segment (arrow). (B) Tc-Dfd expression in a germ band extending embryo as limb buds are just about to form. Expression is present throughout the mandibular and maxillary segments. (C-J) Expression of Tc-Dfd (blue) and Tc-cnc (red) in wild-type embryos. Coexpression of Tc-Dfd and Tc-cnc is brown. (C) Early germ band extending embryo. (D) Germ band extending stage embryo prior to limb bud formation. (E) Germ band extending embryo. (F) Late germ band extending embryo. (G) Same embryo as (F), but a lower plane of focus that shows the reduction of $T c$-cnc expression in the mesoderm (asterisk). (H) Germ band retracting embryo. (I) Embryo undergoing dorsal closure with the gnathal appendages moving towards the ventral midline. (J) Same embryo as (I), but a lower plane of focus that shows the reduction of TC-cnc expression in the mesoderm (asterisk). (C,D) Prior to limb bud formation, Tc-Dfd expression is continuous throughout the mandibular segment. (E,F) As soon as the endites start to form, TCDfd expression retracts from the developing mandibular endites (star) whilst $T c-c n c$ expression is maintained throughout the mandibular appendage. (G-J) By late embryogenesis, faint Tc-Dfd expression is only present in the lateral part of the mandibular limb bud (arrow), and missing from the ventral-medial region (star). Tc-Dfd expression is still strongly maintained in the maxillary limb bud. Mandibular (Mn), maxillary (Mx) and labial (La) segments. 
Some modifications, for example in the frequency and duration of washes, were incorporated from alternative in situ hybridization protocols [45].

Stained embryos were dissected from their yolk and mounted in glycerol. Embryos (and cuticle preparations) were observed using differential interference contrast (DIC) microscopy with an Imager M1 microscope (Carl Zeiss Ltd., Cambridge, UK). Images were taken with Axiocam HRC (Carl Zeiss Ltd., Cambridge, UK) and processed using Axiovision product suite software release 4.8.2 (Carl Zeiss Ltd., Cambridge, UK). Images were edited with GIMP (release 2.6.10.) [46].

\section{Scanning electron microscopy}

Embryos were fixed as described for the whole mount in situ hybridization protocol. Fixed embryos were rinsed in ethanol and immersed in hexamethyldisilazane (HMDS), air dried and sputter coated with gold. Images were taken in a JEOL JSM-5410LV scanning microscope (JEOL Ltd., Tokyo, Japan) at a magnification of 100 to 350 fold and processed with DigitalMicrograph (Gatan Inc., Pleasanton, California, USA).

\section{Results}

\section{Tc-cnc expression}

$T c-c n c$ is expressed in two distinct domains, an anterior cap that includes the developing labrum and around the stomodeum and a posterior collar domain in the mandibular segment (see Figure 1A) [37]. Tc-cnc expression remains constant in these two domains from their first appearance during germ band elongation and through late embryogenesis (see Figure 1D,F,H) and is expressed in regions of the mandibular limb bud where $T c-D f d$ expression becomes repressed (see star in Figure 1E-I). In the mandibular limb bud, Tc-cnc is expressed predominantly in the ectoderm, with weaker expression (or no discernable expression) in the mesoderm of the limb bud (see asterisk in Figure 1G,J).

\section{$T c-D f d$ expression retracts from the developing mandible}

$T c-D f d$ is expressed throughout the mandibular and maxillary segments in the early developing Tribolium embryo (see Figure 1B). As the mandibular limb buds start to form, $T c-D f d$ expression progressively retracts from the ventral-proximal region of the mandibular limb bud (see Figure $1 \mathrm{E}-\mathrm{J}$ ). $T c-D f d$ continues to retract from this ventral-proximal region (star in Figure 1E).

In the developing maxillae, $T c-D f d$ expression is continually expressed in the protopodite (see Figure 1E-J). Mandibular $T c-D f d$ is increasingly repressed until only weak expression remains on the lateral side of the mandible (see Figure 1H,I and Figure 2A,C,D).

The mandibular limb bud has two lobes, the inner and the outer (see Figure 2A). The distal-most part of the mandibular limb bud becomes the outer lobe of the mandible and develops into the future incisor process. Tc-Dfd is not present in this most distal region, which is more clearly noticeable in lateral orientations of dissected Tribolium embryos (see Figure 2C).

We found that $T c-p r d$, in addition to its function as a secondary pair-rule gene [47], is expressed in the predicted location of the developing endites of the embryonic mandibular, maxillary and labial limb buds (see Figure 2B) [48]. We therefore used Tc-prd expression as a marker for endite development. Tc-prd expression reveals that the ventral-medial region of the mandibular limb bud, where $T c-D f d$ expression is lost, encompasses the mandibular endite and the immediate surrounding tissue. $T c-D f d$ expression is retained in the lateral part of the mandibular limb bud, but fades throughout embryogenesis (Figure 2D). Tc-Dfd expression is absent (or considerably weaker) in the distal part of the maxillary palps throughout embryogenesis (see arrow in Figure 2E).

\section{Tc-cnc RNAi phenotype}

In order to test the role $T c-c n c$ might play in patterning the mandibular segment, the gene was knocked down in developing embryos by injecting Tc-cnc dsRNA into female pupae. The knockdown phenotype was determined in the offspring of injected parents using cuticle preparations of their first instar larvae (see Figure 3).

Injection of Tc-cnc dsRNA produces phenotypes that relate to both the cap domain and the collar domain of Tc-cnc expression. The effect in the collar domain is the homeotic transformation of the mandibular appendage into a maxillary identity showing that the posterior collar domain of $T c-c n c$ expression differentiates the mandible from the maxillary appendage. This is shown in Figure 3D,F, where $T c-c n c^{R N A i}$ larvae can be seen to possess an additional pair of maxillae. The mandibular appendages are transformed into a maxillary identity, in possession of a maxillary palp, and maxillary endites (which in wild-type first instar Tribolium larvae are fused to form the ventral branch; see Figure 3A,C). Knockdown of the cap domain results in a dramatic deletion of the labrum showing $T c-c n c$ is necessary to pattern this structure (see Figure 4B). There are also abdominal defects visible in some embryos, although it is possible that this aspect of the phenotype was an artifact of the cuticle preparation procedure.

\section{Tc-cnc represses Tc-DIl and modifies Tc-prd expression in the Mandibular segment}

To investigate the transformed mandibular appendage in Tc-cnc knockdown embryos, the expression patterns of the homeobox genes Tc-prd and Tc-Dll were studied as 


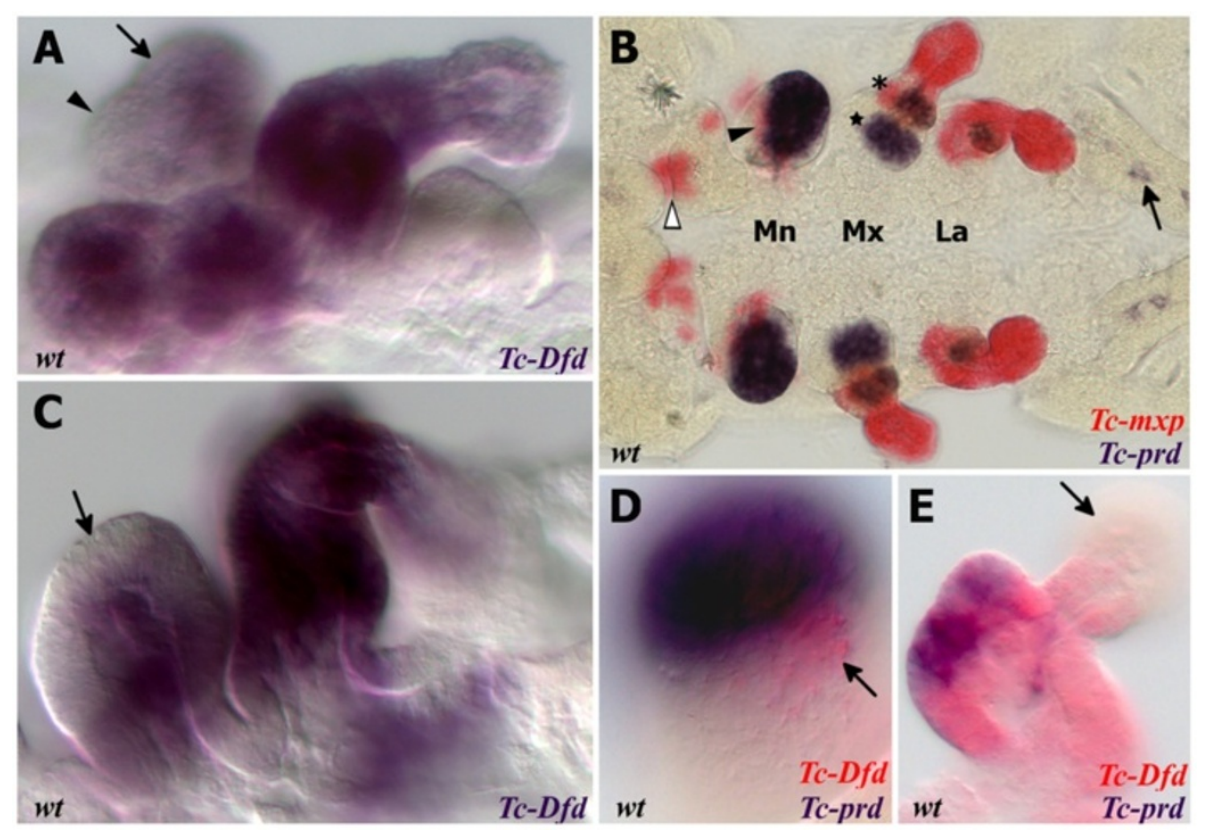

Figure 2 Expression of $T c-D f d, T c-m x p$ and $T c-p r d$ in dissected embryonic mandibles and maxillae. All views are ventral with anterior to the left unless otherwise indicated. Gene expression was determined by in situ hybridization. $(\mathbf{A}, \mathbf{C})$ Lateral view of the mandibular and maxillary appendages showing Tc-Dfd expression (blue) in a germ band fully retracted stage embryo. (A) Tc-Dfd expression is repressed from the developing mandibular endite, which consists of an inner lobe (arrowhead) and an outer lobe (arrow). (B) Embryo stained with $T_{c}$-mxp (red) and Tc-prd (blue). Tc-mxp is expressed in the maxillary and labial palps and the distal protopodite of both appendages. In the maxilla, protopodite expression relates to the position of the developing galea endite lobe (asterisk), which is marked by the distal domain of Tc-prd expression. Tc$m \times p$ is expressed in the mesoderm of the mandibular limb bud (arrowhead). The intercalary domain of Tc-mxp expression is also visible (white arrowhead). Mesodermal expression of Tc-prd is present in the telopodites of post-antennal appendages but clearly visible in the developing leg appendages (arrow). (C) Tc-Dfd expression is missing from the outer lobe of the mandible (arrow). (D,E) Tc-Dfd expression (red) and Tc-prd expression (blue) in a dissected mandible and maxilla of a post germ band retracted stage embryo undergoing dorsal closure. Distal is top. (D) Lateral view of a dissected mandible. Tc-Dfd expression remains on the lateral side of the mandible (arrow). (E) Dissected maxilla, lateral is to the right. Tc-Dfd expression is throughout the protopodite and at the base of the palp. The distal part of the palp is lacking or has weak Tc-Dfd expression (arrow).

genetic markers of the developing endites and telopodites respectively (see Figure 5).

In wild-type embryos, Tc-prd is expressed in the developing endites of all three pairs of gnathal appendages (mandibles, maxillae and labia; see Figure $2 \mathrm{~B}$ and Figure 5A,C). There are two distinct domains of Tc-prd expression in the maxilla, which we assume correspond to the developing lacinia and galea. There is a single domain of Tc-prd in the labial appendage and a larger single domain of expression in the mandibular appendage.

$T c-D l l$ is expressed in the distal part of all appendages of wild-type Tribolium embryos except the mandible. In the developing maxilla, there are two domains of Tc-Dll expression, a distal domain in the developing palp and a proximal domain in the lacinia endite. Tc-cnc RNAi results in homeotic transformation of the mandibular appendage into maxillary identity. The solitary domain of Tc-prd expression in the mandible is transformed into two domains of Tc-prd expression that relate to the maxillary endites (see Figure 5B,D-F). Tc-Dll is de-repressed resulting in expression in the palp and in a proximal endite that appears on the transformed mandible.

The transformed mandibular appendage develops more slowly than the adjacent true maxillary appendages at several stages of embryogenesis resembling the maxillary appendage of an earlier stage (see Figure 5E). By late embryogenesis, there is no evident morphological difference between the maxillae and the ectopic maxillary appendages on the mandibular segment.

Asymmetry of different appendages in Tc-cnc RNAi embryos is often evident in germ band extending stage embryos and occurs left or right at random (see Figure 5E). This does not appear to be an artifact of the RNAi procedure or the in situ hybridization process as appendages other than the mandible can be affected and parental RNAi experiments of other genes in Tribolium have not yielded a similar result (data not shown). Instead this may be related to a loss of the role that $c n c$ has been shown to have in Drosophila in protecting the embryo from oxidative stress [49]. 


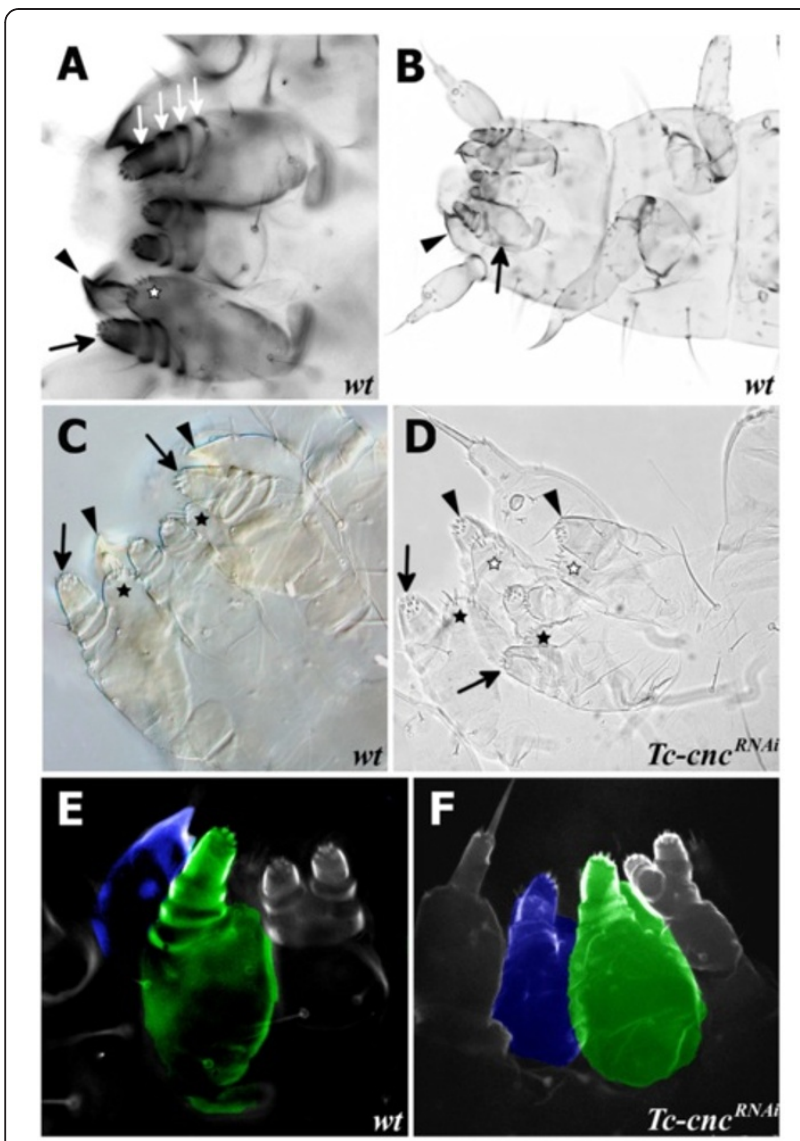

Figure $3 T C-C n C^{R N A i}$ results in transformation of the mandible into maxillary identity. Mandible (arrowhead), maxillary palp (arrow) and maxillary ventral branch (star) are indicated on cuticle

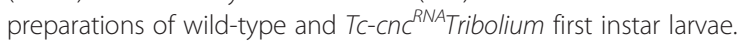
(A) Cuticle preparations of gnathal appendages visualized by fluorescence microscopy. The maxillary appendages have a palp with four segments (white arrows) attached to a protopodite with the maxillary endites (lacinia and galea) that, in first instar larvae, are fused to form the ventral branch (star). (B) Cuticle preparation of a first instar Tribolium larva. (C) Cuticle preparation of the larval gnathal appendages of a wild-type Tribolium larva visualized by DIC microscopy. (D) Cuticle preparation of the gnathal appendages of a $T c-c n c^{R N A i}$ larva. Knockdown of Tc-cnc results in transformation of the mandibular appendages into maxillary appendages (arrowheads). The ventral branch is visible on the transformed appendages (white stars). The maxillary appendage is indicated with arrows (palp) and black stars (ventral branch). (E) Cuticle preparation of wild-type Tribolium larva visualized by confocal microscopy. The mandibular appendage is highlighted in blue; the maxillary appendage is highlighted in green. (F) Cuticle preparation of a Tc-cnc $C^{R N A i}$ larva visualized by confocal microscopy. The transformed mandibular appendage is highlighted in blue and clearly resembles the maxillary appendage (highlighted in green).

Tc-Dfd and Tc-mxp are expressed in a maxilla-like manner in the transformed mandibular limb bud of $T c-c n c^{R N A i}$ embryos

The Hox genes Tc-Dfd and maxillopedia (Tc-mxp), the Tribolium ortholog of $p b$, pattern the maxillary appendage

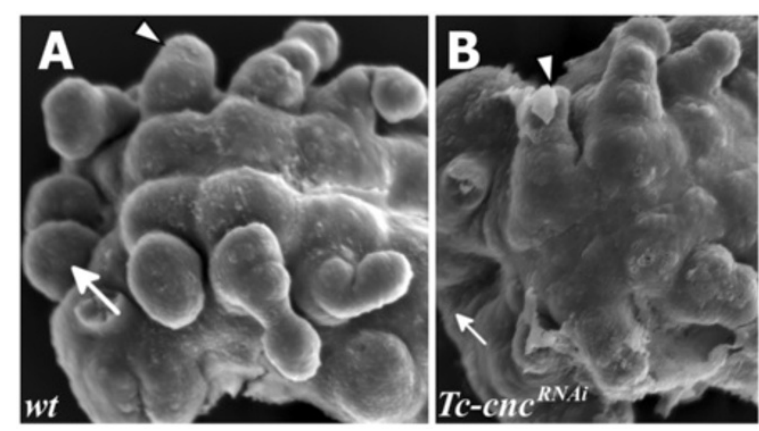

Figure $4 T c-c n c^{R N A i}$ results in deletion of the Labrum. Scanning electron micrographs (SEMs) of wild-type and Tc-cnc ${ }^{R N A}$ embryos shows the deletion of the Labrum in Tc-cnc ${ }^{R N A i}$ embryos. All views are ventral with anterior to the left. (A) SEM of a wild-type embryo at fully extended germ band stage. The labral buds are clearly visible at the anterior of the embryo (arrow). The mandible is indicated (arrowhead). (B) SEM of TC-Cnc ${ }^{R N A i}$ embryo at germ band extending stage. The labral buds are missing (arrow). The mandible is transformed into maxillary identity (arrowhead).

in an additive fashion. $T c-D f d$ is expressed in the proximal part of the maxilla (the protopodite), and $T c-m x p$ is expressed in the palp and is excluded from the proximal part of the protopodite, although it is expressed in the distal protopodite and galea endite (see Figure 2B). Tc-Dfd patterns the protopodite: the proximal part of the appendage including the endite [36]. Tc-mxp patterns the telopodite (the palp) and mutants of $T c-m x p$ possess legs instead of palps in both the maxillary and labial segments. These transformed appendages are attached to a protopodite that is unaffected by the loss of $T c-\operatorname{mxp}[50,51]$.

As Tc-cnc RNAi results in a homeotic transformation of the mandible into a maxilla, we predicted that both the Hox genes responsible for patterning the maxillary appendage will be expressed in the maxillary pattern in the homeotically transformed appendage. It was found that this is indeed the case (see Figure 6).

In wild-type embryos, $T c-D f d$ expression retracts from the mandibular limb bud (see Figure 6C,F). In $T c-c n c^{R N A i}$ embryos, the mandible is transformed to maxillary identity and $T c-D f d$ expression is retained in the protopodite of this transformed appendage (see Figure 6D,G-I).

Tc-mxp is expressed in the maxillary and labial palps in wild-type embryos (see Figure 6A,C,F). In the maxillae, $T c$ $m x p$ is expressed in the distal part of the protopodite, including the galea endite. In the transformed mandibular appendage of $T c-c n c^{R N A i}$ embryos, $T c-m x p$ is expressed in the ectoderm of the ectopic palp as it is in the maxillary palp and also includes expression in the galea endite and distal protopodite (see Figure 6B,D,E,G,I).

$T c-m x p$ is expressed in the mesoderm of the mandibular appendages of wild-type embryos (white arrow in Figure 6A,F) [51]. Interestingly, this mesodermal expression of $T c-m x p$ is seen in $T c-c n c$ knockdown embryos 


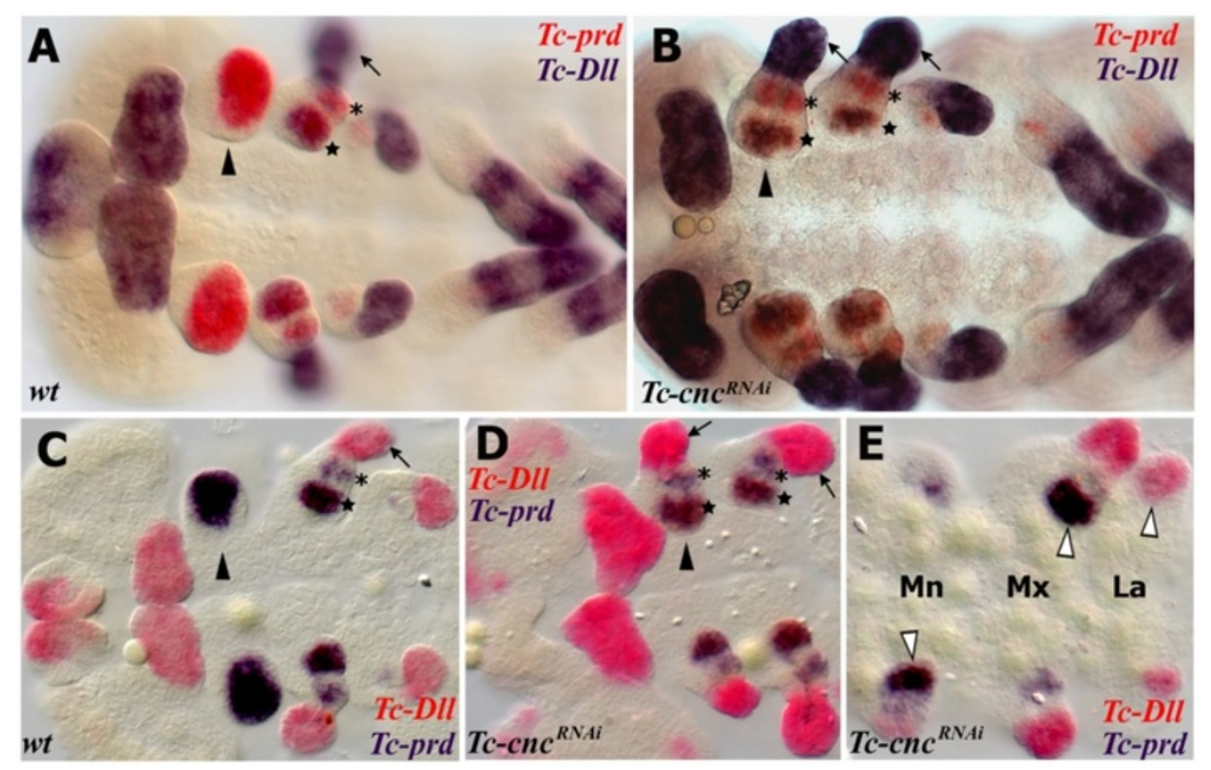

Figure 5 Homeotic transformation of the mandibular appendage to maxillary identity in Tc-cnc knockdown embryos as revealed by the expression of markers for telopodites (Tc-DII) and endites (Tc-prd). Gene expression was determined by in situ hybridization. All views are ventral with anterior to the left. (A-E) The mandibular segment appendage (arrowhead), lacinia (star), galea (asterisk) and telopodite (arrow) are indicated. (A,B) Expression of Tc-D/l (blue) and Tc-prd (red). (A) wild-type embryo. Tc-D/l is expressed in the maxillary lacinia endite lobe (star) and telopodite (arrow). Tc-prd is expressed in the endites of the mandible, maxilla and labial appendages. (B) Tc-cnc $C^{R N A i}$ embryo. Tc-DIl and Tc-prd are expressed in transformed mandible appendages in the same manner as in the maxillae. The labral domain of Tc-D/l is also missing at the anterior of the embryo. (C-E) Expression of Tc-D/l (red) and Tc-prd (blue) in germ band extending embryos earlier than those shown in $\mathbf{A}, \mathbf{B}$. (C) wild-type embryo. (D,E) TC-CnC ${ }^{R N A i}$ embryos. (E) TC-CnC $C^{R N A i}$ embryo. The telopodites and endites of some appendages are larger (white arrowheads) than the corresponding appendage on the other side of the same segment. There is an asymmetry between the different transformed mandibular appendages. The transformed mandibles resemble maxillae at an earlier stage of development and so have delayed development relative to the maxillary appendages. Mandibular (Mn), maxillary (Mx) and labial (La) segments shown.

(white arrow in Figure 6E,H). This suggests that there is $c n c$ independent regulation of Tc-mxp in the mandibular limb bud. Tc-cnc is expressed in the ectoderm of the mandibular limb bud, and expression is weaker (or absent) in the mesoderm.

\section{Tc-Dfd activates the posterior 'collar' domain of Tc-cnc in the mandibular segment}

Experiments performed on Drosophila have shown that $D f d$ does not activate $c n c$ expression [52]. In order to investigate whether $T c-D f d$ has any role in regulating $T c$ cnc expression in Tribolium, we knocked down Tc-Dfd by parental RNAi and detected $T c-c n c$ expression via in situ hybridization.

Surprisingly, we found that in $T c-D f d^{R N A i}$ embryos the posterior collar domain of $T c-c n c$ expression is completely missing from all stages of embryo investigated, from germ band extending embryos through to stages where embryos are undergoing dorsal closure (Figure 7). The anterior cap domain of expression is unaffected. This shows that, unlike in Drosophila, Tc-Dfd is necessary for the activation of the posterior domain of $T c-c n c$ in the mandibular segment of Tribolium.

\section{Tc-Dfd activates Tc-prd expression in the mandible and maxillary segments}

Brown et al. have shown that $T c-D f d$ is required to pattern the mandible and the proximal part of the maxillary appendages In $T c$ - $D f d$ mutants, the mandible is transformed to antennal identity and the maxillae lose the endites whilst retaining the palp [36].

In order to further investigate the role of $T c-D f d$ in patterning the gnathal appendages, we studied Tc-prd expression in $T c-D f d^{R N A i}$ knockdown embryos. In $T c-D f d^{R N A i}$ knockdown embryos, Tc-prd expression is lacking in both the transformed mandible (ectopic antennae) and the affected maxillary appendages (see Figure 8C,D). Tc-prd is still expressed in the developing labial endite. This result shows that $T c-D f d$ is necessary for the activation of $T c-p r d$ expression in the mandibular and maxillary segments and is further evidence that $T c-D f d$ is required for development of the endites on these segments.

\section{Discussion}

The role of Tc-cnc in patterning the mandible of Tribolium We sought to understand mandible patterning in a model arthropod that has a mandible with primitive characteristics. Our results show that $T c-c n c$ is required 


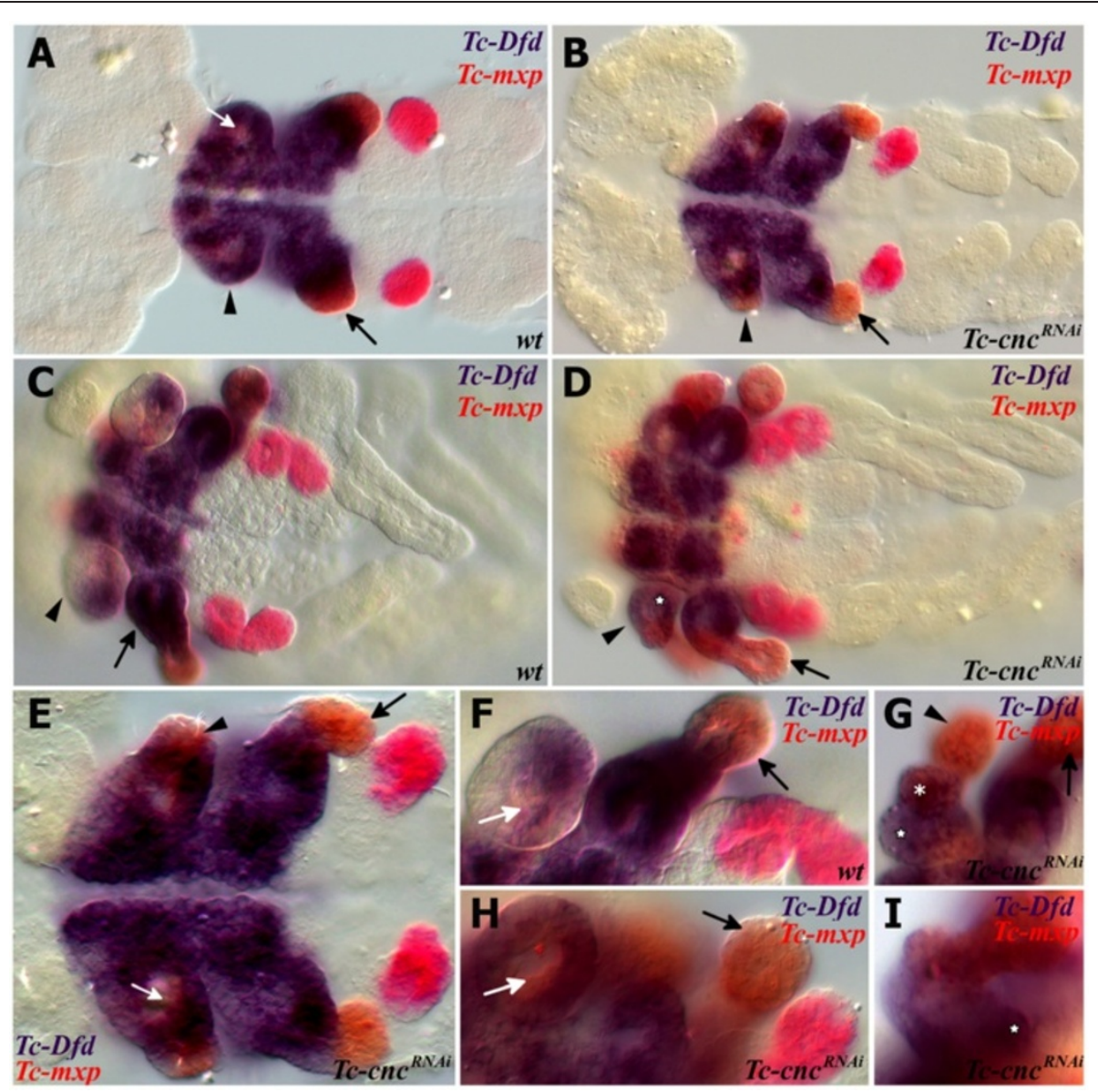

Figure 6 Expression of the Hox genes Tc-Dfd and Tc-mxp in wild-type and Tc-cnc ${ }^{R N A i}$ embryos. Knockdown of Tc-cnc by RNAi results in transformation of the mandibular appendage to maxillary identity and the expression of Hox genes in a similar manner to that seen in the maxilla. All views are ventral with anterior to the left. Expression of Tc-Dfd (blue) and Tc-mxp (red) was determined by in situ hybridization. Mandibular segment is indicated with an arrowhead, maxillary segment with a black arrow. Mesodermal expression of $T c-m \times p$ is indicated with a white arrow. (A,C,F) Wild-type Tribolium embryos. (B,D,E,G-I) Tc-cnc ${ }^{R N A i}$ embryos. (A) Wild type germ band extending embryo. Tc-mxp is expressed in the developing maxillary and labial palps and the mesoderm in the mandibular segment (white arrow). (B) TC-CnC ${ }^{R N A i}$ germ band extending embryo: $T c-m \times p$ expression is present in the transformed mandibular appendage (arrowhead) in a telopodite domain consistent with the transformation of the mandible to maxillary identity. (C) Wild-type germ band retracting stage embryo. Tc-Dfd expression has retracted from the majority of the mandibular appendage. (D) Tc-cnc ${ }^{R N A i}$ embryo at a similar stage to C. Tc-Dfd expression is present in the transformed mandibular protopodite (star). Tc-mxp is expressed in the transformed mandibular appendage palp. (E) Higher magnification of the earlier germ band extending stage $T c-c n C^{R N A i}$ embryo shown in B. (F) Higher magnification of the gnathal appendages of a germ band retracting stage at a similar stage to $\mathbf{C}$. $(\mathbf{G}, \mathbf{H}, \mathbf{I})$ Higher magnification of the gnathal appendages of germ band retracting stage $T c-c n c^{R N A i}$ embryos. (G) Tc-Dfd is expressed throughout the transformed mandibular appendage, in the lacinia endite (star) and galea endite (asterisk). Tc-mxp is expressed in the palp (arrowhead) as well as the galea endite in a manner that is identical to the maxilla (arrow). (H) The mesodermal expression domain of $T c-$ mxp (white arrow) is observed in the transformed mandibular appendage. (I) Tc-Dfd is expressed throughout the maxilla, the rounded kink at the base of the maxilla is indicated (star).

for specification of the identity of the mandibular segment of Tribolium and differentiates the mandible from a maxilla.

Knockdown of Tc-cnc transcripts by parental RNAi results in a homeotic transformation of the mandible into maxillary identity in Tribolium embryos and first instar larvae. The homeotic transformation is also evident in the changed expression of the genes Tc-Dll and Tc-prd (markers for the developing telopodite and endite of the maxilla) in knockdown embryos.
The Hox genes $T c-m x p$ and $T c-D f d$ are required to pattern the maxillary appendage and do so in an additive manner, $T c-D f d$ patterns the base of the appendage and $T c-m x p$ patterns the palp [36,51]. We show that in Tc-cnc knockdown embryos, $T c-D f d$ and $T c-m x p$ are expressed in a maxilla like pattern in the transformed mandibular appendage.

We show that the 'collar' domain of Tc-cnc in the mandibular segment is activated by $T c-D f d$ in Tribolium. The mandibular segment collar domain of $c n c$ is not activated or regulated by $D f d$ or by any other Hox gene in 

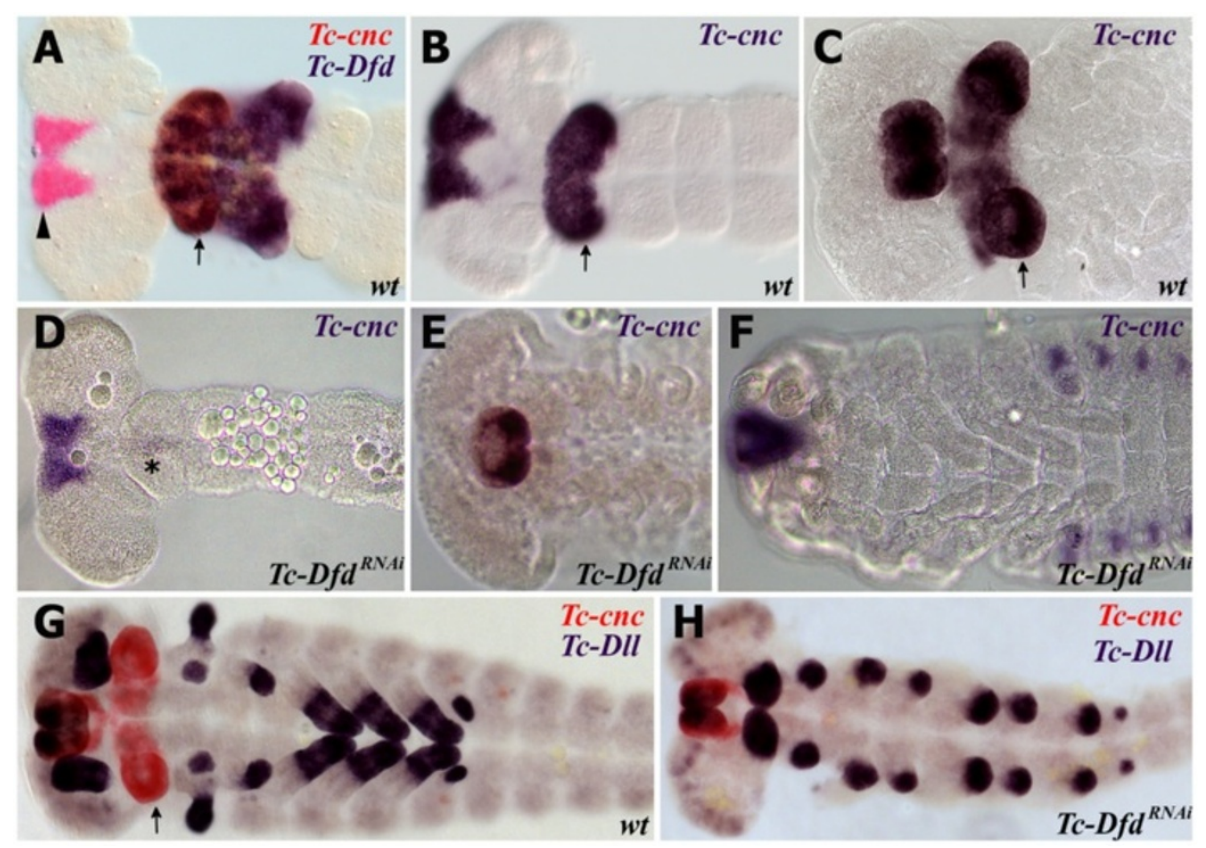

Figure 7 Tc-Dfd activates the posterior collar domain of Tc-cnc in the mandibular segment. Gene expression was determined by in situ hybridization. (2A-C) Tc-cnc expression in wild-type embryos. Throughout embryogenesis, Tc-cnc expression consists of an anterior cap domain in the labrum (arrowhead) and a collar domain (arrow) in the mandibular segment. (A) Tc-cnc (red) and Tc-Dfd (blue) expression in a germ band extending embryo. (B) Tc-cnc expression (blue) in a germ band extending embryo at a similar but slightly earlier stage to (A). (C) Tc-cnc expression (blue) in later stage embryo prior to dorsal closure. (D-F) Tc-cnc expression in Tc-Dfd ${ }^{\text {RNAi }}$ embryos. In all stages, from germ band extending (D), germ band retracted (E) and during dorsal closure (F), the posterior domain of TC-Cnc is missing in the mandibular segment, whilst the anterior domain of $T c-c n c$ is expressed as normal showing that $T c-c n c$ is activated by $T c-D f d$ in the mandibular segment. There is a faint stripe of Tc-cnc in the mandibular segment of (D) (asterisk), this may be due to partial knockdown effects. ( G) Expression of Tc-cnc (red) and Tc-D/l (blue) in wild-type germ band retracting embryo.( $\mathbf{H})$ Expression of Tc-cnc (red) and Tc-D/l (blue) in a Tc-Dfd ${ }^{R N A i}$ germ band extending embryo. The posterior domain of Tc-Cnc is missing.

Drosophila [52]. We also show that $T c-D f d$ is necessary for the expression of Tc-prd in both the mandible and the maxilla.

Based upon the results of this and previous studies we present a model for the roles of these genes in mandible patterning in Tribolium (see Figure 9).

\section{The role of Tc-cnc in patterning the labrum of Tribolium}

The deletion of the labrum in $T c-c n c^{R N A i}$ embryos is consistent with the loss of the cap domain of $T c-c n c$ expression in the labrum. The labrum is a structure of considerable interest as it is shared by all extant groups of euarthropods whilst its evolution and development remain controversial. The labrum has appendage-like characteristics and may have evolved from a fused pair of appendages, for example from structures homologous to the anterior antennae of lobopods [53]. However, unlike all other paired arthropod appendages, the labrum is not associated with a segment and may have a different origin [54].

\section{Comparisons with Drosophila}

There are many similarities between Tribolium and Drosophila in the expression patterns of genes in the mandibular and maxillary segments and in how these segments are patterned. In both insects $D f d$ and $c n c$ are both required to pattern the mandibular segment. $c n c$ is required for the patterning of labral derived structures and the differentiation of the mandible from maxillary identity. chc represses $\mathrm{Dll}$ expression in the mandibular segment.

The Hox genes $D f d$ and $p b / T c-m x p$ are also expressed in similar proximal and distal domains respectively in the maxillary segment limb bud or gnathal lobe as are prd and Dll. Dfd patterns proximal structures that are derived from the maxillary lobe or limb buds. In both species, $D f d$ activates the proximal domain of $D l l$ $[24,25,36]$. Tc-Dfd activates the maxillary prd domain in both Drosophila, and also, as we have shown in this study, in Tribolium [55,56].

There are nevertheless differences in the patterning of the mandibular and maxillary segments between Tribolium and Drosophila. In Drosophila, loss of cnc function does not result in a full homeotic transformation of the mandibular gnathal lobe to maxillary identity, rather, the mandibular gnathal lobe is transformed into just the proximal part of the maxillary gnathal lobe [24]. This is 


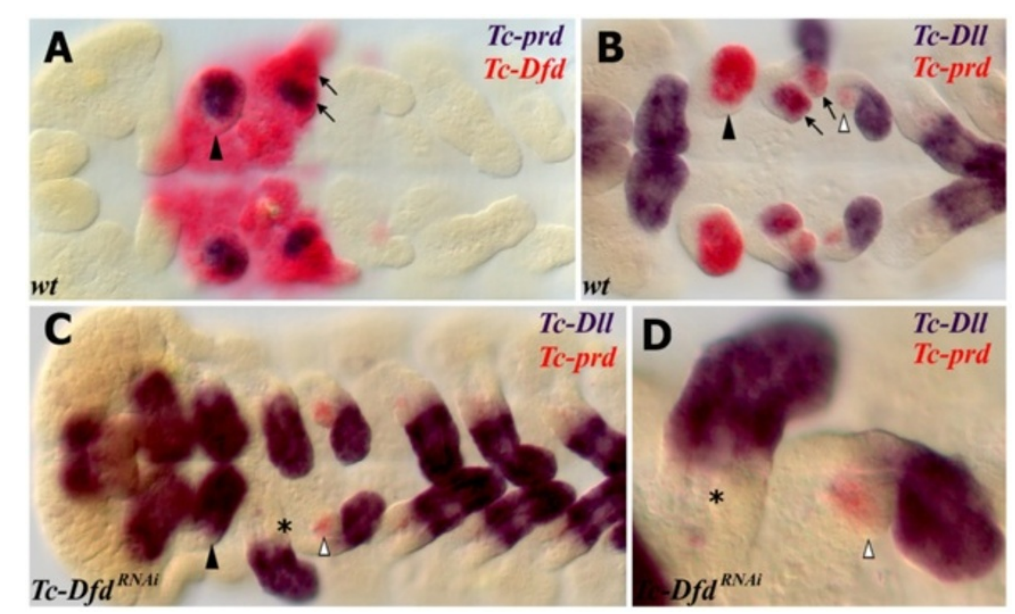

Figure $8 T c$-Dfd knockdown results in the loss of Tc-prd expression in the embryonic mandibular and maxillary segments. Tc-Dfd patterns the endites of the mandibular and maxillary segments. The mandibular segment appendage is marked with an arrowhead. The maxillary endites are marked with arrows in wild-type embryos $(\mathbf{A}, \mathbf{B})$. The labial endites are marked with white arrowheads. (A) Expression of Tc-prd (blue) and Tc-Dfd (red) in a wild-type germ band extending embryo. Tc-prd is expressed in the developing endites of the mandible and maxilla. Tc-Dfd is expressed in the mandibular and maxillary segments. (B) Expression of Tc-prd (red) and Tc-D/l (blue) in a wild-type germ band extending embryo. Tc-prd is expressed in the mandible, maxillary and labial appendages. Tc-D/l is expressed in the lacinea endite lobe. There is no Tc-D/l expression in the mandible (arrowhead). (C,D) Expression of Tc-prd (red) and Tc-DIl (blue) in a Tc-Dfd ${ }^{R N A i}$ germ band extending embryo. (C) The mandible has been transformed into an ectopic antenna, which expresses Tc-D/l (arrowhead) and lacks Tc-prd expression. There is no endite and no Tc-prd expression (asterisk) in the maxilla. The labial appendage has an endite (white arrowhead) marked with Tc-prd expression. (D) Enlargement of the maxilla and labial appendage shown in (C).

in contrast to Tribolium where loss of Tc-cnc function results in a complete transformation of mandible to maxillary identity.

In addition to the activation of $T c-c n c$ in the mandibular segment by $T c-D f d$, another difference between Drosophila and Tribolium is the regulation of collier ( $\mathrm{col}$ ) by cnc. The anterior mandibular expression of $c n c$ is upstream of col in Drosophila and both genes are required to pattern the hypopharyngeal lobes [57-59]. In Tribolium, which does not have hypopharyngeal lobes, it has been recently shown that $T c-c n c$ is not activated by $T c$ col [60].

\section{The role of $c n c$ as a repressor of maxilla patterning Hox genes}

While we have shown that Tc-cnc patterns the mandible and differentiates the mandible from a maxilla, the precise role that it has in patterning the mandibular segment is not clear. The many similarities in the patterning function of $c n c$ in Tribolium and Drosophila suggest that the molecular functions of $\mathrm{Cnc}$ protein revealed by experiments in Drosophila may be similar in Tribolium.

Research in Drosophila has demonstrated the role of $c n c$ as a repressor of Hox gene function in the mandibular segment $[23,24]$. $c n c$ has been shown to repress $D f d$ transcription and Dfd protein activity in the anterior mandibular segment in Drosophila [23,24]. There is coexpression of $c n c$ and $D f d$ in the posterior of the mandibular segment, indicating that some mandibular expression of $D f d$ is not affected by the presence of $c n c$ $[23,24]$. Dfd has also been shown to repress $p b$ in the ectoderm of the mandibular segment in Drosophila [61].

As the dynamics of Tc-Dfd expression in Tribolium resemble the dynamics of Dfd expression in Drosophila, with initial coexpression followed by subsequent repression of $T c-D f d$ in a part of the mandibular segment, it seems likely that a similar situation is occurring in Tribolium.

We have shown that $T c-c n c$ is necessary for both the repression of $T c-D f d$ expression in the mandibular limb bud and the repression of the ectodermal palp domain of Tc-mxp in the developing mandibular limb bud. However, further research is needed to determine whether $T c$ - $c n c$ has a direct functional role in the repression of these Hox genes.

\section{The possible role of $T c$-cnc as a direct activator of} mandible patterning genes

In Drosophila, several lines of evidence suggest that Cnc functions as an activator, activating mandibular segment specific patterning genes and thereby indirectly repressing Hox genes [23]. cnc also patterns some mandibular segment derived structures independently of $D f d$. Ectopic activation of $c n c$ in Drosophila embryos results in ectopic hypopharyngeal lobe derived structures [23]. Although the hypopharyngeal lobes have been thought to derive from the intercalary segment, it has recently been 


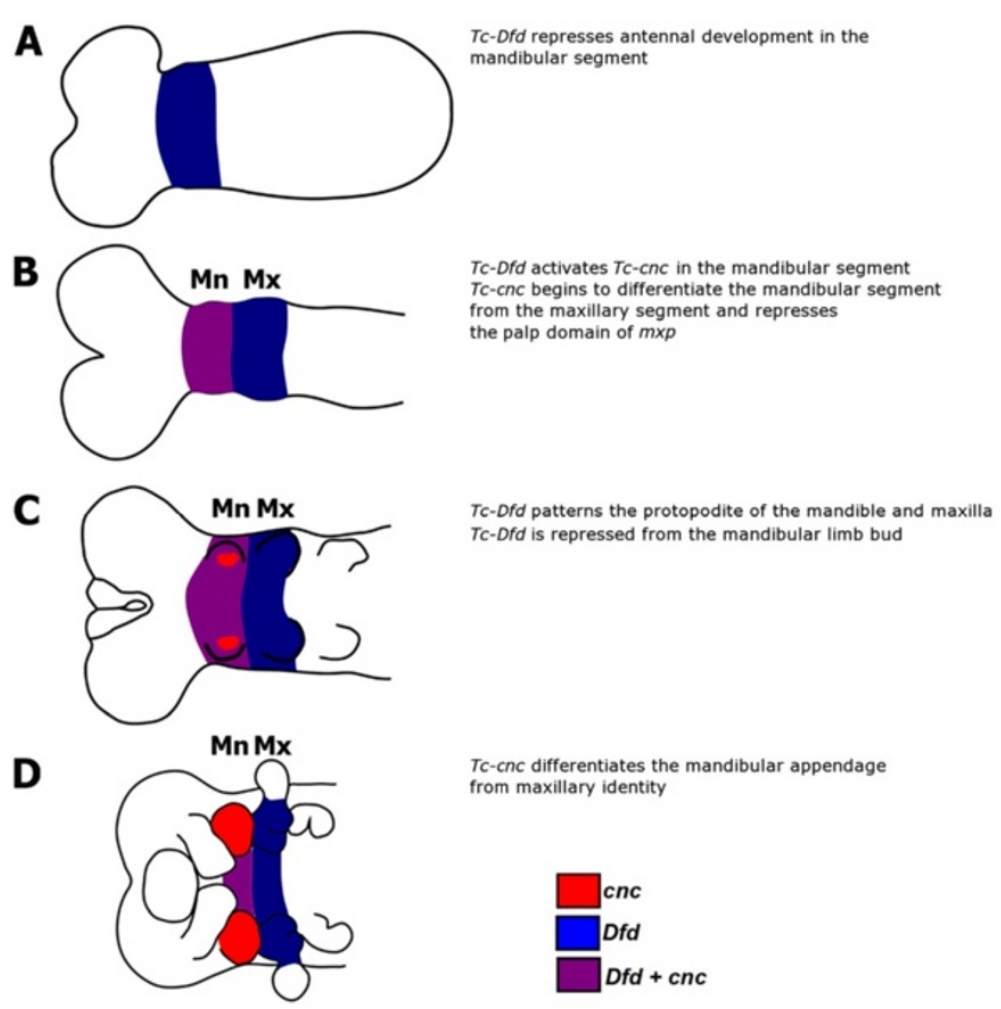

Figure $9 \mathrm{~A}$ model of $T c-c n c$ and $T c-D f d$ mandibular and maxillary patterning functions in Tribolium castaneum. Tc-Dfd patterns both the mandible and maxillary segments. Tc-Dfd patterns the protopodites of these appendages. Note that the anterior 'cap' domain of Tc-cnc has been omitted from this scheme for clarity. Tc-Dfd expression is shown in blue, Tc-cnc expression is shown in red. Tc-cnc and Tc-Dfd expression is shown in purple. (A) Tc-Dfd is expressed in the mandibular and maxillary segments and patterns these segments. In the mandibular segment, $T c-D f d$ represses antennal development. Tc-Dfd patterns the maxillary segment in conjunction with Tc-mxp. (B) Tc-Dfd activates Tc-cnc expression and together Tc-cnc and Tc-Dfd cooperate to pattern the mandibular segment. (C) Tc-Dfd patterns the protopodite of the mandibular and maxillary appendages in germ band extending embryos, but is repressed from the mandibular limb bud as it develops. (D) In germ band retracting stage embryos, Tc-cnc has differentiated the mandibular appendage from maxillary identity.

shown that they are in fact derived from the mandibular segment [37]. This result indicates that $c n c$ is in fact necessary and sufficient to pattern some mandibular segment derived structures suggesting that $T c-c n c$ may directly activate mandible patterning genes in Tribolium.

\section{Conserved expression of $c n c$, Dfd and $p b$ in mandibulate arthropods}

Comparison of the expression of $c n c$ homologs in mandibulates suggests that both functions of the labral patterning anterior 'cap' domain and the mandible patterning posterior 'collar' domain are conserved in mandibulate arthropods. Species that have been studied in addition to Drosophila and Tribolium include the cricket Acheta domestica, the milkweed bug Oncopeltus fasciatus, and the firebrat Thermobia domestica [39,40]. Outside insects, only one species has been studied to date, the myriapod Glomeris marginata, which also shows expression in a cap and a mandibular collar [38].

The expression patterns of orthologs of $D f d$ and $p b$ are also conserved in other mandibulates suggesting that patterning of the maxilla may also be conserved. $D f d$ is expressed in the mandible and maxilla bearing segments in the majority of mandibulates and expression is stronger in the protopodite than in the palps of maxillary appendages [36,39,62-66]. There is loss of $D f d$ expression in the mandibular limb bud across mandibulates, as in Tribolium and Drosophila $[24,35,65,66]$. Expression of $p b$ is conserved in the telopodites of these maxillary appendages $[39,65,67]$.

In an onychophoran, the closest extant outgroup to the Arthropoda, a homolog of $D f d$ is expressed in the proximal region of each walking limb bud [68] suggesting that $D f d$ expression in the base of the mandibular and maxillary limbs may be the primitive condition in the Arthropoda.

cnc and the evolution of the mandible from a maxilla-like precursor

The manner in which $c n c$ differentiates the mandible from maxillary identity may ultimately provide clues about how the mandible has evolved from a maxilla-like 
precursor in the stem lineage of mandibulate arthropods.

A study of the fossil record shows that the mandible has evolved from a particular type of jointed appendage, the biramous limb (see Figure 10A). In the ancestor to the arthropods, the primitive post-antennal limbs were similar in structure [12]. As stem lineage arthropods diverged during the Cambrian, post-antennal biramous limbs diverged from the primitive biramous limb structure. The likely precursor to the mandible was a maxilla-like appendage, with numerous well-defined endites similar to those present on other post-antennal segments (see Figure 10G). Such a maxilla-like second post-antennal limb is present in numerous 'crustaceamorph' stem lineage mandibulate arthropods like Martinssonia elongata and the Phospatocopida $[2,11,69,70]$.

We hypothesize that, in the stem lineage to the mandibulate arthropods, $D f d$ patterned the base of the ancestral monopodial limb (see Figure 10B) and the protopodite of the primitive biramous gnathal appendages (see Figure 10C). At some point in the stem- lineage leading to the mandibulate arthropods, cnc acquired a new role patterning the mandibular segment: differentiating the mandibular endite and protopodite from those of the maxilla resulting in the mandibular gnathal edge (see Figure 10D).

The mandible has probably evolved from a biramous maxilla-like precursor by modification of the most proximal endite to form the characteristic mandibular gnathal edge whilst, at least primitively, retaining both the telopodite palp and the exopodite (see Figure 10G).

\section{The role of $c$ c homologs in chelicerates and onychophorans}

To test the idea that the function of $c n c$ evolved to pattern the mandible in the lineage leading to the mandibulates, it is necessary to study $c n c$ homologs in outgroups to the Mandibulata with the prediction that it does not have a comparable role in patterning the segment homologous to the mandibular segment (the first leg segment in chelicerates).

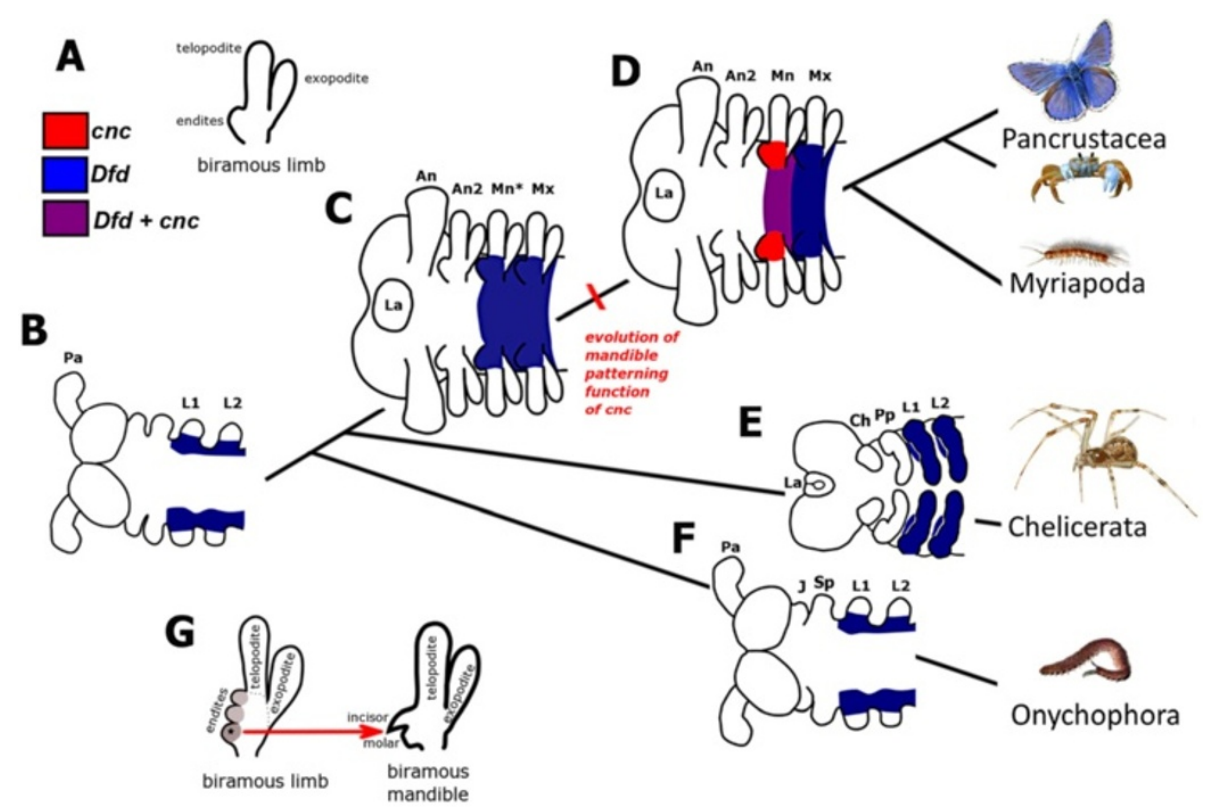

Figure 10 Hypothetical evolution of the mandible patterning function of $c n c$ in embryos in the stem lineage of the mandibulate arthropods. (A) The post-antennal limbs of stem-lineage mandibulates are serially homologous biramous limbs with multiple endites, represented here as a single lobe for clarity, on the medial part of the protopodite. (B) Hypothetical expression of Dfd in a lobopod, the ancestor to all arthropods (and closely related taxa such as the Onychophora) based on expression of Dfd in an onychophoran [68]. Here, Dfd is expressed proximally in monopodial limbs with the anterior limit at the segment homologous to the first leg segment (L1) of chelicerates and onychophorans. (C) Hypothetical expression of Dfd in a hypothetical non-mandibulate ancestor to Mandibulata. (D) Expression of $c n c$ and Dfd in a hypothetical ancestor to the mandibulate arthropods (Pancrustacea and Myriapoda). The mandibular segment identity is specified by cnc. We hypothesize that the mandible patterning function of $\mathrm{cnc}$ evolved in the stem lineage of the mandibulate arthropods. The mandibular and maxillary segments of mandibulates are homologous to the first and second leg segments of chelicerates and onychophorans. (E) Expression of Dfd in chelicerates based upon Dfd expression in spiders. There are two homologs of Dfd in spiders, both of which are expressed in the L1 to L4 segments. (F) Expression of Dfd in an onychophoran. (G) The mandibular gnathal edge, consisting of an incisor and molar, most likely evolved from the proximal endite (star) on the primitive biramous limb present in species such as Martinssonia. The other more distal endites were lost at some point. Labels are: antenna (An), first leg (L1), jaws (J), labrum (La), mandible (Mn), maxilla (Mx), maxilla-like mandible precursor (Mn*), primary antenna (Pa), second antenna (An2), second leg (L2), slime papilla (Sp). 
The homologous segment to the mandibular segment in the chelicerates and the onychophorans is the first leg segment and homologs of $D f d$ are expressed in this segment (see Figure 10E,F) $[4,5,68]$. In these groups there is no obvious differentiation between the first leg appendage and the second leg appendage (maxilla homolog). It is therefore not obvious what role a 'collar' domain of $c n c$ would perform in chelicerates or onychophorans.

Although the expression of $c n c$ is not known in nonmandibulate arthropods, expression of chelicerate anterior Hox genes such as $D f d$ and $p b$ are different in several respects to the conserved expression of these genes in mandibulate arthropods [62]. This suggests that the conserved expression of Hox genes in the mouthparts of the mandibulate arthropods is a synapomorphy for the Mandibulata $[5,71,72]$.

The closest related outgroup of the Mandibulata in which a $c n c$ homolog has been investigated is the nematode Caenorhabditis elegans. The C. elegans cnc homo$\log$, Skn1, has been shown to have developmental role in patterning mesoderm and endoderm derived structures $[73,74]$. One important, non-developmental role of $c n c$ (and its homologs across Bilateria) that has been studied in some detail is its role in xenobiotic and oxidative stress responses [49,75-77]. This role has been discovered in diverse organisms and is likely to be present both in mandibulates and in closely related outgroups to the Mandibulata such as the chelicerates.

\section{Conclusions}

Our study is the first functional investigation of some of the genes necessary specifically to pattern the mandible of an arthropod species with a canonical mandible in which the gnathal edge is made up of the incisor and molar processes.

Using parental RNAi to knockdown gene transcripts in Tribolium, we show that $T c-c n c$ is required for specification of the identity of the mandibular appendage and differentiates it from maxillary identity. Analysis of gene expression by in situ hybridization shows that Tc-cnc is required for the repression of the maxillary expression domains of the Hox genes $T c-m x p$ and $T c-D f d$, which pattern the maxilla. We also show that Tc-cnc is necessary for the formation of the labrum. The mandible differentiating function of $T c-c n c$ is similar to the role of cnc in Drosophila in patterning the mandibular segment; in both beetle and fly, $c n c$ and $D f d$ cooperate to specify mandibular identity. One significant difference is that $T c-c n c$ is activated by $T c-D f d$ in the mandibular segment in Tribolium whereas $c n c$ is activated independently of Dfd in Drosophila.

Similar expression patterns of $c n c, D f d$ and $p b$ homologs in other mandibulate arthropods suggests that the functions of these genes are conserved, that $c n c$ also differentiates the mandible from the maxilla in these species and that $c n c$ evolved a mandible patterning function in the lineage leading to the mandibulates and possibly acts in conjunction with $D f d$ to achieve this.

To show that $c n c$ has a conserved role in patterning the mandible across Mandibulata requires study of the function of $c n c$, or at the very least additional expression data, in more representatives of the mandibulate arthropods. In particular, expression data are lacking from any crustacean species.

\section{Competing interests}

The authors declare that they have no competing interests.

\section{Authors' contributions}

JFC and MJT conceived and designed the study. JFC collected the data and JFC and MJT analyzed the results. JFC and MJT drafted the manuscript and approved the final manuscript for submission.

\section{Acknowledgements}

The authors would like to thank Gregor Bucher (Göttingen) for providing a culture of Tribolium castaneum beetles. This work was supported by funding from the Biotechnology and Biological Sciences Research Council (BBSRC).

Received: 25 June 2012 Accepted: 23 August 2012

Published: 1 November 2012

\section{References}

1. Edgecombe GD, Richter S, Wilson GDF: The mandibular gnathal edges: homologous structures throughout Mandibulata? Afr Invertebr 2003, 44:115-135.

2. Edgecombe GD: Arthropod phylogeny: an overview from the perspectives of morphology, molecular data and the fossil record. Arthropod Struct Dev 2010, 39:74-87.

3. Kraus O: "Myriapoda" and the ancestry of the Hexapoda. Ann Soc Entomol (N S) 2001, 37:105-127.

4. Telford MJ, Thomas RH: Expression of homeobox genes shows chelicerate arthropods retain their deutocerebral segment. Proc Natl Acad Sci USA 1998, 95:10671-10675.

5. Damen WGM, Hausdorf M, Seyfarth EA, Tautz D: A conserved mode of head segmentation in arthropods revealed by the expression pattern of Hox genes in a spider. Proc Natl Acad Sci USA 1998, 95:10665-10670.

6. Janssen R, Eriksson BJ, Budd GE, Akam M, Prpic NM: Gene expression patterns in an onychophoran reveal that regionalization predates limb segmentation in pan-arthropods. Evol Dev 2010, 12:363-372.

7. Mayer $G$, Whitington PM: Velvet worm development links myriapods with chelicerates. Proc Biol Sci 2009, 276:3571-3579.

8. Regier JC, Shultz JW, Ganley AR, Hussey A, Shi D, Ball B, Zwick A, Stajich JE, Cummings MP, Martin JW, Cunningham CW: Resolving arthropod phylogeny: exploring phylogenetic signal within $41 \mathrm{~kb}$ of protein-coding nuclear gene sequence. Syst Biol 2008, 57:920-938.

9. Rota-Stabelli O, Telford MJ: A multi criterion approach for the selection of optimal outgroups in phylogeny: recovering some support for Mandibulata over Myriochelata using mitogenomics. Mol Phylogenet Evol 2008, 48:103-111.

10. Regier JC, Shultz JW, Zwick A, Hussey A, Ball B, Wetzer R, Martin JW, Cunningham CW: Arthropod relationships revealed by phylogenomic analysis of nuclear protein-coding sequences. Nature 2010, 463:1079-1083.

11. Rota-Stabelli O, Campbell L, Brinkmann H, Edgecombe GD, Longhorn SJ, Peterson KJ, Pisani D, Philippe H, Telford MJ: A congruent solution to arthropod phylogeny: phylogenomics, microRNAs and morphology support monophyletic Mandibulata. Proc Biol Sci 2011, 278:298-306.

12. Boxshall GA: The evolution of arthropod limbs. Biol Rev Camb Philos Soc 2004, 79:253-300.

13. Chen JY: The sudden appearance of diverse animal body plans during the Cambrian explosion. Int J Dev Biol 2009, 53:733-751. 
14. Waloszek D, Maas A, Chen JY, Stein M: Evolution of cephalic feeding structures and the phylogeny of Arthropoda. Palaeogeogr Palaeoclimatol Palaeoevol 2007, 254:273-287.

15. Niwa N, Saitoh M, Ohuchi H, Yoshioka H, Noji S: Correlation between Distal-less expression patterns and structures of appendages in development of the two-spotted cricket, Gryllus bimaculatus. Zool Sci 1997, 14:115-125.

16. Scholtz G, Mittmann B, Gerberding M: The pattern of Distal-less expression in the mouthparts of crustaceans, myriapods and insects: new evidence for a gnathobasic mandible and the common origin of Mandibulata. Int J Dev Biol 1998, 42:801-810.

17. Popadic A, Panganiban G, Rusch D, Shear WA, Kaufman TC: Molecular evidence for the gnathobasic derivation of arthropod mandibles and for the appendicular origin of the labrum and other structures. Dev Genes Evol 1998, 208:142-150.

18. Chadwick R, Jones B, Jack T, McGinnis W: Ectopic expression from the Deformed gene triggers a dominant defect in Drosophila adult head development. Dev Biol 1990, 141:130-140.

19. Abzhanov A, Holtzman S, Kaufman TC: The Drosophila proboscis is specified by two Hox genes, proboscipedia and Sex combs reduced, via repression of leg and antennal appendage genes. Development 2001, 128:2803-2814.

20. Joulia L, Bourbon HM, Cribbs DL: Homeotic proboscipedia function modulates hedgehog-mediated organizer activity to pattern adult Drosophila mouthparts. Dev Biol 2005, 278:496-510.

21. Joulia L, Deutsch J, Bourbon HM, Cribbs DL: The specification of a highly derived arthropod appendage, the Drosophila labial palps, requires the joint action of selectors and signaling pathways. Dev Genes Evol 2006, 216:431-442.

22. Mohler J, Mahaffey JP, Deutsch E, Vani K: Control of Drosophila head segment identity by the bZIP homeotic gene cnc. Development 1995 , 121:237-247.

23. Veraksa A, McGinnis N, Li X, Mohler J, McGinnis W: Cap 'n' collar B cooperates with a small Maf subunit to specify pharyngeal development and suppress deformed homeotic function in the Drosophila head. Development 2000, 127:4023-4037.

24. McGinnis N, Ragnhildstveit E, Veraksa A, McGinnis W: A cap ' $n$ ' collar protein isoform contains a selective Hox repressor function. Development 1998, 125:4553-4564

25. Regulski M, McGinnis N, Chadwick R, McGinnis W: Developmental and molecular analysis of Deformed; a homeotic gene controlling Drosophila head development. EMBO J 1987, 6:767-777.

26. Angelini DR, Kaufman TC: Functional analyses in the hemipteran Oncopeltus fasciatus reveal conserved and derived aspects of appendage patterning in insects. Dev Biol 2004, 271:306-321.

27. Hughes CL, Kaufman TC: RNAi analysis of Deformed, proboscipedia and Sex combs reduced in the milkweed bug Oncopeltus fasciatus: novel roles for Hox genes in the hemipteran head. Development 2000, 127:3683-3694.

28. Jurgens G, Lehmann R, Schardin M, Nusslein-Volhard C: Segmental organisation of the head in the embryo of Drosophila melanogaster: a blastoderm fate map of the cuticle structures of the larval head. Roux's Arch Dev Biol 1986, 1986:359-377.

29. Schinko JB, Kreuzer N, Offen N, Posnien N, Wimmer EA, Bucher G: Divergent functions of orthodenticle, empty spiracles and buttonhead in early head patterning of the beetle Tribolium castaneum (Coleoptera). Dev Biol 2008, 317:600-613.

30. Finkelstein R, Perrimon N: The molecular genetics of head development in Drosophila melanogaster. Development 1991, 112:899-912.

31. Rogers BT, Kaufman TC: Structure of the insect head in ontogeny and phylogeny: a view from Drosophila. Int Rev Cytol 1997, 174:1-84.

32. Merrill VK, Turner FR, Kaufman TC: A genetic and developmental analysis of mutations in the Deformed locus in Drosophila melanogaster. Dev Biol 1987, 122:379-395

33. O'Hara E, Cohen B, Cohen SM, McGinnis W: Distal-less is a downstream gene of Deformed required for ventral maxillary identity. Development 1993, 117:847-856.

34. Mohler J, Vani K, Leung S, Epstein A: Segmentally restricted, cephalic expression of a leucine zipper gene during Drosophila embryogenesis. Mech Dev 1991, 34:3-9.

35. Brown SJ, Holtzman S, Kaufman TC, Denell RE: Characterization of the Tribolium Deformed ortholog and its ability to directly regulate Deformed target genes in the rescue of a Drosophila Deformed null mutant. Dev Genes Evol 1999, 209:389-398.

36. Brown SJ, DeCamillis MA, Gonzalez-Charneco K, Denell M, Beeman RW, Nie W, Denell RE: Implications of the Tribolium Deformed mutant phenotype for the evolution of Hox gene function. Proc Natl Acad Sci USA 2000, 97:4510-4514.

37. Economou AD, Telford MJ: Comparative gene expression in the heads of Drosophila melanogaster and Tribolium castaneum and the segmental affinity of the Drosophila hypopharyngeal lobes. Evol Dev 2009, 11:88-96.

38. Janssen R, Budd GE, Damen WGM: Gene expression suggests conserved mechanisms patterning the heads of insects and myriapods. Dev Biol 2011, 357:64-72.

39. Rogers BT, Peterson MD, Kaufman TC: The development and evolution of insect mouthparts as revealed by the expression patterns of gnathocephalic genes. Evol Dev 2002, 4:96-110.

40. Birkan M, Schaeper ND, Chipman AD: Early patterning and blastodermal fate map of the head in the milkweed bug Oncopeltus fasciatus. Evol Dev 2011, 13:436-447.

41. Bucher G, Scholten J, Klingler M: Parental RNAi in Tribolium (Coleoptera). Curr Biol 2002, 12:R85-R86.

42. Brown SJ, Mahaffey JP, Lorenzen MD, Denell RE, Mahaffey JW: Using RNA to investigate orthologous homeotic gene function during development of distantly related insects. Evol Dev 1999, 1:11-15.

43. Wohlfrom H, Schinko JB, Klingler M, Bucher G: Maintenance of segment and appendage primordia by the Tribolium gene knodel. Mech Dev 2006, 123:430-439.

44. Schinko JB, Posnien N, Kittelmann S, Koniszewski N, Bucher G: Single and double whole-mount in situ hybridization in red flour beetle (Tribolium) embryos. Cold Spring Harb Protoc 2009, 2009:prot5258. pdb.

45. Kosman D, Mizutani CM, Lemons D, Cox WG, McGinnis W, Bier E: Multiplex detection of RNA expression in Drosophila embryos. Science 2004, 305:846

46. GIMP: The GNU Image Manipulation Program. http://www.gimp.org.

47. Choe CP, Miller SC, Brown SJ: A pair-rule gene circuit defines segments sequentially in the short-germ insect Tribolium castaneum. Proc Natl Acad Sci USA 2006, 103:6560-6564.

48. Aranda M, Marques-Souza H, Bayer T, Tautz D: The role of the segmentation gene hairy in Tribolium. Dev Genes Evol 2008, 218:465-477.

49. Grimberg KB, Beskow A, Lundin D, Davis MM, Young P: Basic leucine zipper protein $\mathrm{Cnc}-\mathrm{C}$ is a substrate and transcriptional regulator of the Drosophila 26S proteasome. Mol Cell Biol 2011, 31:897-909.

50. Shippy TD, Brown SJ, Denell RE: Maxillopedia is the Tribolium ortholog of proboscipedia. Evol Dev 2000, 2:145-151

51. Shippy TD, Guo J, Brown SJ, Beeman RW, Denell RE: Analysis of maxillopedia expression pattern and larval cuticular phenotype in wildtype and mutant Tribolium. Genetics 2000, 155:721-731.

52. Mohler J: Genetic regulation of CNC expression in the pharyngeal primordia of Drosophila blastoderm embryos. Roux's Arch Dev Biol 1993, 202:214-223.

53. Budd GE, Telford MJ: The origin and evolution of arthropods. Nature 2009, 457:812-817.

54. Posnien N, Bashasab F, Bucher G: The insect upper lip (labrum) is a nonsegmental appendage-like structure. Evol Dev 2009, 11:480-488.

55. Gutjahr T, Frei E, Noll M: Complex regulation of early paired expression: initial activation by gap genes and pattern modulation by pair-rule genes. Development 1993, 117:609-623.

56. Li X, Murre C, McGinnis W: Activity regulation of a Hox protein and a role for the homeodomain in inhibiting transcriptional activation. EMBO J 1999, 18:198-211.

57. Crozatier M, Valle D, Dubois L, Ibnsouda S, Vincent A: Collier, a novel regulator of Drosophila head development, is expressed in a single mitotic domain. Curr Biol 1996, 6:707-718.

58. Crozatier M, Valle D, Dubois L, Ibnsouda S, Vincent A: Head versus trunk patterning in the Drosophila embryo; collier requirement for formation of the intercalary segment. Development 1999, 126:4385-4394.

59. Seecoomar M, Agarwal S, Vani K, Yang G, Mohler J: Knot is required for the hypopharyngeal lobe and its derivatives in the Drosophila embryo. Mech Dev 2000, 91:209-215.

60. Schaeper ND, Pechmann M, Damen WGM, Prpic NM, Wimmer EA: Evolutionary plasticity of collier function in head development of diverse arthropods. Dev Biol 2010, 344:363-376. 
61. Rusch DB, Kaufman TC: Regulation of proboscipedia in Drosophila by homeotic selector genes. Genetics 2000, 156:183-194.

62. Hughes $C L$, Kaufman TC: Hox genes and the evolution of the arthropod body plan. Evol Dev 2002, 4:459-499.

63. Kokubo H, Ueno K, Amanai K, Suzuki Y: Involvement of the Bombyx Scr gene in development of the embryonic silk gland. Dev Biol 1997, 186:46-57.

64. Walldorf $U$, Binner P, Fleig R: Hox genes in the honey bee Apis mellifera. Dev Genes Evol 2000, 210:483-492.

65. Hughes $\mathrm{CL}$, Kaufman TC: Exploring the myriapod body plan: expression patterns of the ten Hox genes in a centipede. Development 2002, 129:1225-1238.

66. Janssen R, Damen WGM: The ten Hox genes of the millipede Glomeris marginata. Dev Genes Evol 2006, 216:451-465.

67. Abzhanov A, Kaufman TC: Homeotic genes and the arthropod head: expression patterns of the labial, proboscipedia, and Deformed genes in crustaceans and insects. Proc Natl Acad Sci USA 1999, 96:10224-10229.

68. Eriksson BJ, Tait NN, Budd GE, Janssen R, Akam M: Head patterning and Hox gene expression in an onychophoran and its implications for the arthropod head problem. Dev Genes Evol 2010, 220:117-122.

69. Muller KJ, Waloszek D: Martinssonia elongata gen. et sp. n., a crustaceanlike euarthropod from the upper cambrian 'Orsten'. Zool Scrip 1986, 15:73-92.

70. Siveter DJ, Williams M, Waloszek D: A phosphatocopid crustacean with appendages from the Lower Cambrian. Science 2001, 293:479-481.

71. Abzhanov A, Popadic A, Kaufman TC: Chelicerate Hox genes and the homology of arthropod segments. Evol Dev 1999, 1:77-89.

72. Schwager EE, Schoppmeier M, Pechmann M, Damen WGM: Duplicated Hox genes in the spider Cupiennius salei. Front Zool 2007, 4:10.

73. Bowerman B, Eaton BA, Priess JR: Skn-1, a maternally expressed gene required to specify the fate of ventral blastomeres in the early $C$. elegans embryo. Cell 1992, 68:1061-1075.

74. Walker AK, See R, Batchelder C, Kophengnavong T, Gronniger JT, Shi Y, Blackwell TK: A conserved transcription motif suggesting functional parallels between Caenorhabditis elegans SKN-1 and Cap'n'Collar-related basic leucine zipper proteins. J Biol Chem 2000, 275:22166-22171.

75. Motohashi H, Yamamoto M: Nrf2-Keap1 defines a physiologically important stress response mechanism. Trends Mol Med 2004, 10:549-557.

76. Sykiotis GP, Bohmann D: Stress-activated cap'n'collar transcription factors in aging and human disease. Sci Signal 2010, 3:re3.

77. Misra JR, Horner MA, Lam G, Thummel CS: Transcriptional regulation of xenobiotic detoxification in Drosophila. Genes Dev 2011, 25:1796-1806.

doi:10.1186/2041-9139-3-25

Cite this article as: Coulcher and Telford: Cap'n'collar differentiates the mandible from the maxilla in the beetle Tribolium castaneum. EvoDevo 2012 3:25.

\section{Submit your next manuscript to BioMed Central and take full advantage of:}

- Convenient online submission

- Thorough peer review

- No space constraints or color figure charges

- Immediate publication on acceptance

- Inclusion in PubMed, CAS, Scopus and Google Scholar

- Research which is freely available for redistribution 